\title{
INFLUENCE OF SELECTED PARAMETERS ON EDDY CURRENTS ATTENUATION IN NON-DESTRUCTIVE INSPECTION
}

Eddy currents attenuation in conductive materials under non-destructive inspection is analyzed in the paper. Influence of selected parameters of the inspection on the attenuation is studied by numerical means. A circular driving coil is employed to induce eddy currents in conductive plates under harmonic excitation. Outer diameter of the coil is varied together with the clearance between the coil and a plate surface for two plates with different thickness. Attenuation of the eddy current density in plates is studied and the results are analyzed in absolute values and the normalized ones as well. It is demonstrated that the investigated parameters of the inspection have significant impact on the attenuation.

Keywords: Non-destructive evaluation, eddy currents, attenuation, penetration depth.

\section{Introduction}

Eddy current testing (ECT) is one of the most common electromagnetic methods utilized in non-destructive evaluation of conductive materials. The principle of ECT underlies in the interaction of induced eddy currents with a structure of an examined body [1], [2]. A primary alternating exciting electromagnetic field is generated in the vicinity of a coil driven by a time-varying current according to the Ampere's law. Electromotive force is induced in a conductive object which is in proximity of the coil according to the Faraday's law. Eddy-currents flow in the conductive object according to the Ohm's law and their vector lines must be closed. A secondary electromagnetic field generated by the eddy-currents counterworks to the primary exciting electromagnetic field according to the Lenz's theorem. The induction coupling therefore exists between the coil and the conductive object. It can be simply considered as an interaction between the primary and the secondary electromagnetic fields. ECT can thus be employed for detection of surface and subsurface non-homogeneities, measurements of material thickness as well as of the electromagnetic parameters and so on [1]. However, the most wide spread area of its application in present is the detection of discontinuities [2].

ECT probes are one of the most important elements in the non-destructive testing, because they transfer information between an ECT instrument and a conductive object through the induction coupling. Optimal ECT probe should assure high sensitivity to expected defects, high probability of detection of expected defects and classification possibility of expected defects (location, dimensions, etc.) [3]. Exciting coil(s) of ECT probe should thus induce eddy currents with high density and such distribution that eddy current lines are significantly perturbed when a defect is in pres- ence. The detection circuit should assure that maximum of the perturbation field is sensed. Many ECT probes have been developed over past decades reflecting special demands of particular applications. Probe design and development is still of high interest because the area of ECT utilization is gradually wide-spreading [4]-[7].

Eddy current distribution in an inspected material significantly determines basic features of the probe such as sensitivity, penetration and resolution. To be able to detect expected flaws with high probability in a material under given test conditions it is very indispensable to have a knowledge about the eddy current distribution. Many probes have been developed and designed mainly based on practical experiences. Maturity of the numerical means make it possible to build up systematic approach in this sense.

According to the electromagnetic field theory, the distribution of eddy currents along material depth depends mainly on a testing frequency and the electromagnetic parameters of a material as it is given by the well known equation for the standard depth of penetration:

$$
\delta=\frac{1}{\sqrt{\pi f \mu \sigma}}
$$

where $\delta[\mathrm{m}]$ is the standard depth of penetration, $f[\mathrm{~Hz}]$ is the inspection frequency, $\mu\left[\mathrm{H} \cdot \mathrm{m}^{-1}\right]$ and $\sigma\left[\mathrm{S} \cdot \mathrm{m}^{-1}\right]$ are the magnetic permeability and the electric conductivity of the inspected material, respectively. However, under real conditions there are much more parameters with substantial influence on this distribution such as shape of coils, their dimensions, configuration of inspection, material thickness, lift-off, etc [8].

\footnotetext{
* Ladislav Janousek

Department of Electromagnetic and Biomedical Engineering, Faculty of Electrical Engineering, University of Zilina, Zilina, Slovak Republic, E-mail: ladislav.janousek@fel.uniza.sk
} 
The paper numerically analyzes influences of selected parameters of ECT inspection on attenuation of eddy currents in a conductive material.

\section{Simulation Model}

Numerical simulations using the finite element method are carried out to investigate influences of certain inspection parameters on the eddy currents attenuation.

A plate conductive specimen, shown in Fig. 1, is modeled in this study to explore attenuation of eddy currents along its depth. The plate thickness is adjusted to two values $t=10$ and $30 \mathrm{~mm}$ to explore its influence on the attenuation. The electromagnetic parameters of the plate are adjusted to the following values $\sigma=$ $=1.35 \cdot 10^{6} \mathrm{~S} \cdot \mathrm{m}^{-1} \mu_{r}=1$ that correspond to a material SUS316L frequently used for structural components in petrochemical and nuclear industries.

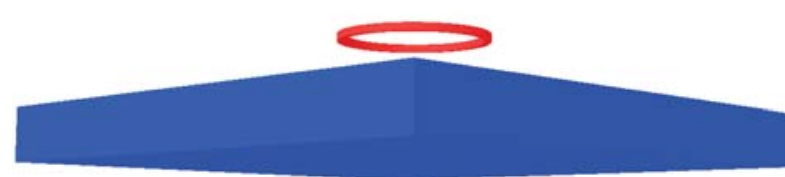

Fig. 1 Layout of model

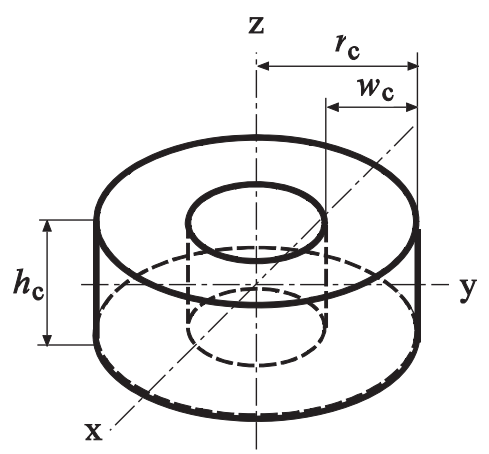

Fig. 2 Layout of circular exciting coil

A circular exciting coil, displayed in Fig. 2, is employed to drive the eddy currents in the plates. Axis of the coil is oriented normally regarding the plate s' surface. This type of exciting coil in the given orientation is commonly utilized in variety of applications because an ECT probe with such exciting coil does not account for the directional properties. Cross-section of the coil winding is kept constant for all the calculations, in concrete $w_{c}=$ $=1 \mathrm{~mm}, h_{c}=1 \mathrm{~mm}$ (see Fig. 2), because these dimensions have only minor impact on the eddy current attenuation along the material depth [9]. The coil radius rc is varied to investigate its influence on the attenuation. It is gradually adjusted to the following values $r_{c}=2,5,10,15,20 \mathrm{~mm}$. Clearance between the coil and the plate surface, hereinafter referred to as the lift-off, is sequentially tuned to $l_{f}=0.5,1,2,5,10 \mathrm{~mm}$. The coil is driven with the harmonic current while the current density is kept constant at a value of $1 \mathrm{~A} \cdot \mathrm{mm}^{-2}$ and the frequency is adjusted to $10 \mathrm{kHz}$. The standard depth of penetration for the given parameters equals to $\delta=4.33 \mathrm{~mm}$. Another three values of the exciting frequency 1,5 and $20 \mathrm{kHz}$ are also used for the investigations for the plate with a thickness of $10 \mathrm{~mm}$.

Very fine model of the system is built up based on the finite tetrahedral elements. Only $1 / 4$ of the system, as shown in Fig. 1, is considered employing appropriate boundary conditions. The mesh consists of approximately 4 million elements. Results of numerical simulations are summarized in the following section.

\section{Results and discussions}

Three parameters of the inspection, i.e. the coil radius $r_{c}$, the lift-off $l_{f}$ and the plate thickness $t$ are varied to investigate influences of these parameters on the eddy current attenuation along the material depth. Important findings are reported and discussed here.

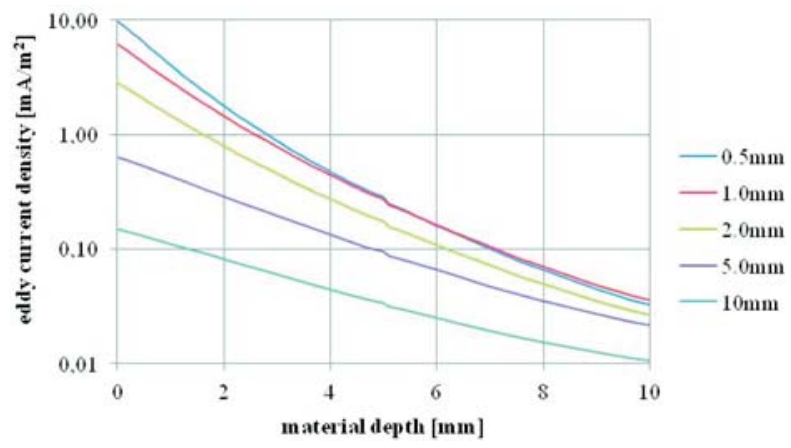

a) plate thickness $t=10 \mathrm{~mm}$

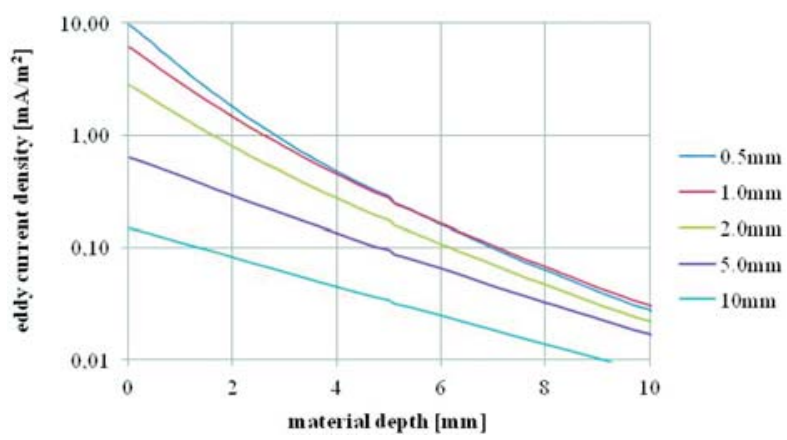

b) plate thickness $t=30 \mathrm{~mm}$

Fig. 3 Dependences of eddy current density in absolute values on the material depth for different adjustments of lift-off, $r_{c}=2 \mathrm{~mm}$ 
The distribution of eddy current density vector in the material is stored after successful execution of each simulation. The results are then processed in such a way that only one dependence of the eddy current density absolute value along the material depth is taken for the evaluation. The dependence is taken along the plate thickness direction (see Fig. 1) under a certain surface position where the eddy current density has the maximum value.

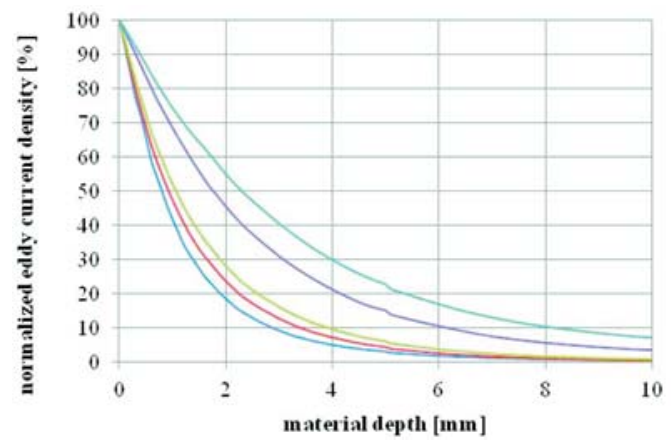

a) plate thickness $t=10 \mathrm{~mm}$

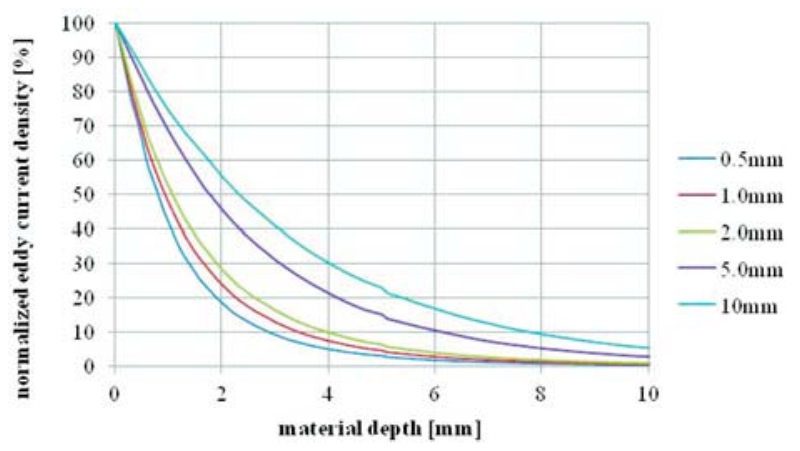

b) plate thickness $t=30 \mathrm{~mm}$

Fig. 4 Dependences of eddy current density in normalized values on the material depth for different adjustments of lift-off, $r_{c}=2 \mathrm{~mm}$

The dependences of the eddy current density absolute value on the material depth for the coil with a radius of $r_{c}=2 \mathrm{~mm}$ and for several values of the lift-off are shown in Fig. 3. The exciting frequency is adjusted to $10 \mathrm{kHz}$. The dependences are shown for the two considered values of the plate thickness. Note that the vertical axis is in logarithmic scale. The upper limit of $\mathrm{x}$-axes for both the plate thicknesses are set to $10 \mathrm{~mm}$ so one can simply compare the results. Attenuation of eddy currents along the material depth has the exponential character. However, it can be observed that the results gained for different values of the lift-off show diverse behaviour. Moreover, it can be seen that the plate thickness almost does not have impact on the attenuation for the given standard depth of penetration. The same dependences but in normalized values are shown in Fig. 4 in order to highlight contrast between the curves.
Each of dependences of the eddy current density absolute value on the material depth is normalized by its maximum value. It can be seen that the eddy current attenuation strongly depends not only on the standard depth of penetration, i.e. the frequency, the conductivity and the permeability, but also on the lift-off, however it does not depend on the material thickness. Larger clearance between the coil and the plate surface provides eddy current distribution with less attenuation along the material depth.

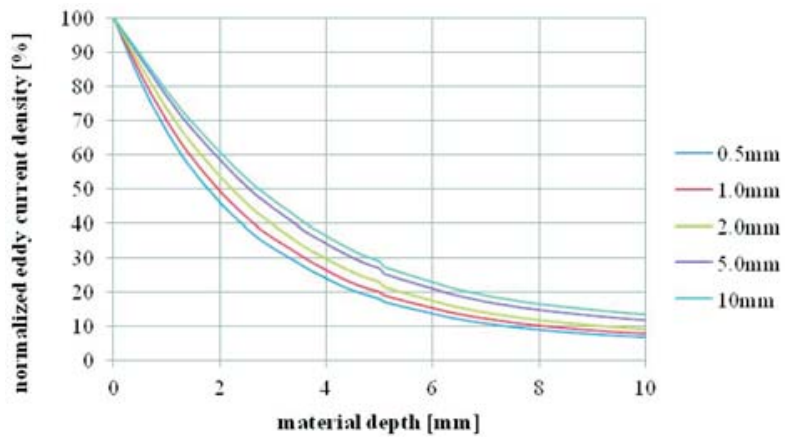

a) plate thickness $t=10 \mathrm{~mm}$

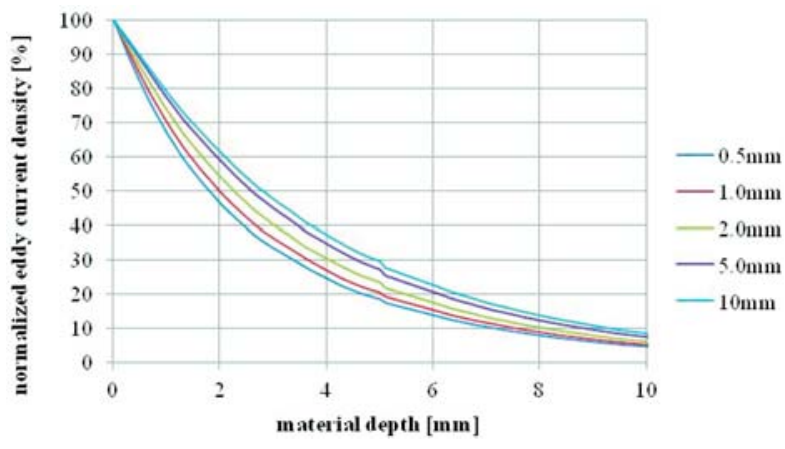

b) plate thickness $t=30 \mathrm{~mm}$

Fig. 5 Dependences of eddy current density in normalized values on the material depth for different adjustments of lift-off, $r_{c}=20 \mathrm{~mm}$

Similar dependences in normalized values for the coil with a radius of $r_{c}=20 \mathrm{~mm}$ are shown in Fig. 5. The presented results clearly show that the impact of the lift-off on the eddy current attenuation along the material depth decreases for the coil with larger diameters. It can be also noticed that the eddy currents are slightly less attenuated in the $10 \mathrm{~mm}$ thick plate comparing to the one with a thickness of $30 \mathrm{~mm}$; however, this impact is not significant.

A specific value of the distance from material surface along its depth, denoted hereinafter as the penetration depth, is calculated for all the evaluated distributions of the eddy current density. It is the value where the eddy current density falls to $50 \%$ of its surface 
value. The penetration depth is then plotted as a dependence on the coil radius rc and the lift-off lf as well. Fig. 6 shows the calculated dependence only for the plate with a thickness of $t=10 \mathrm{~mm}$ as the results presented above showed that the plate thickness does not have significant impact on the attenuation under the frequency of $f=10 \mathrm{kHz}$. As it can be seen, by proper adjustment of the exciting system parameters, the penetration depth can be increased several times comparing to a case of only a small coil placed with a small lift-off over a material.

The eddy current attenuation for the plate with a thickness of $t=10 \mathrm{~mm}$ is investigated also under frequencies $f=1.5$ and $20 \mathrm{kHz}$. Table 1 reports values of the standard penetration depth $\delta$ for the given frequencies calculated according to (1) together with a ratio of the plate thickness ( $t=10 \mathrm{~mm}$ in this case) and the standard depth of penetration $t / \delta$.

The penetration depth, as defined above, as dependence on the coils radius rc and on the lift-off for another considered values of the frequency are shown in Figs. 7-9. It can be observed that the parameters have strong impact on the penetration depth especially when the value of material thickness is comparable to the standard
Standard depth of penetration and its relation

Table 1 to material thickness $\mathrm{t}=10 \mathrm{~mm}$

\begin{tabular}{|c|c|c|c|c|}
\hline$f[\mathrm{kHz}]$ & 1 & 5 & 10 & 20 \\
\hline$\delta[\mathrm{mm}]$ & 13.70 & 6.13 & 4.33 & 3.06 \\
\hline$t / \delta[-]$ & 0.73 & 1.63 & 2.31 & 3.27 \\
\hline
\end{tabular}

depth of penetration. Importance of the parameters decreases with increasing the ratio of the material thickness and the standard depth of penetration

The exciting coil radius and the lift-off have significant influence on the eddy current attenuation along a material depth when the material thickness is at maximum double comparing to the standard penetration depth. It should be noted that in such a case larger coils and/or larger lift-off provide deeper penetration of eddy currents inside a material and thus it would provide better resolution. However, sensitivity can be decreased. Sophisticated design of the exciting system requires therefore appropriate compromise to reach essential performance of an ECT probe.

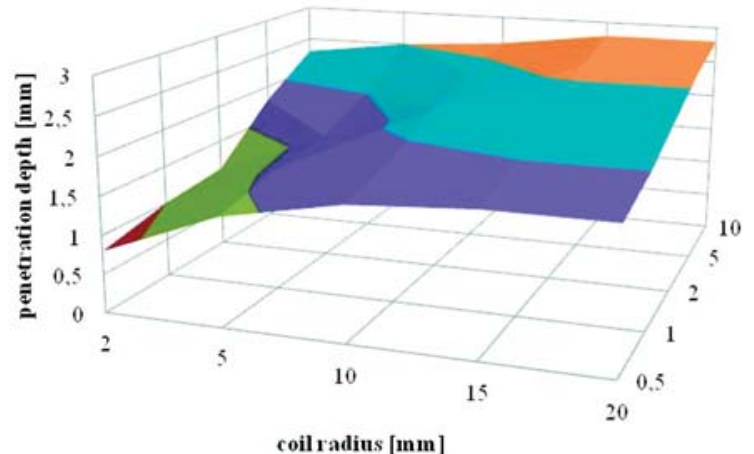

Fig. 6 Penetration depth as function of coil radius and lift-off, $t=10$ $m m, f=10 \mathrm{kHz}$

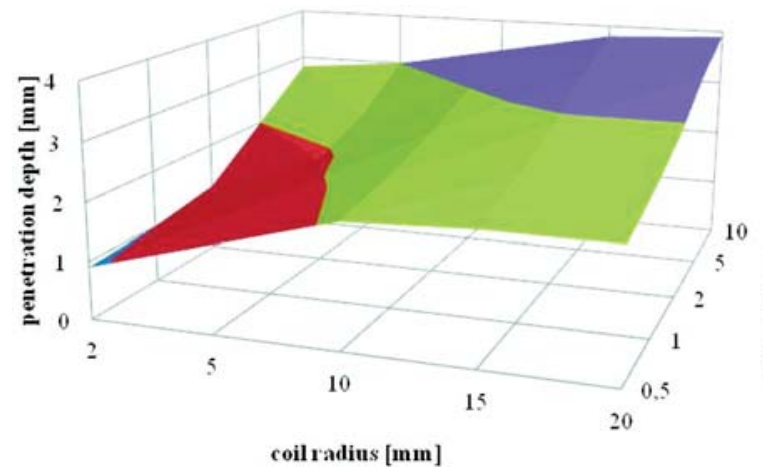

Fig. 8 Penetration depth as function of coil radius and lift-off, $t=10$ $m m, f=5 \mathrm{kHz}$

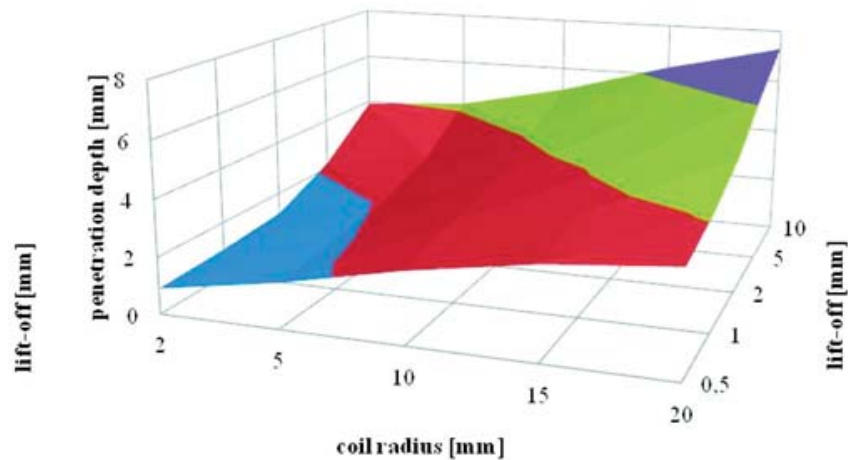

Fig. 7 Penetration depth as function of coil radius and lift-off, $t=10$ $m m, f=1 \mathrm{kHz}$

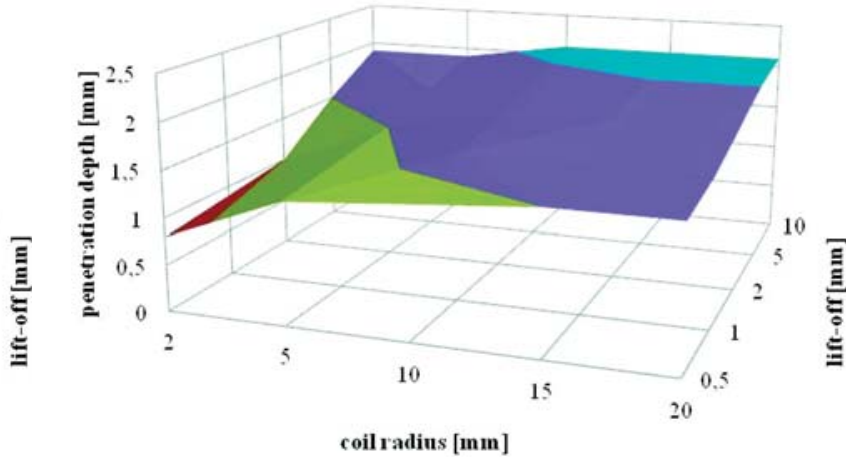

Fig. 9 Penetration depth as function of coil radius and lift-off, $t=10$ $m m, f=20 \mathrm{kHz}$ 


\section{Conclusions}

The paper concerned on attenuation of eddy currents along material depth in non-destructive inspection. Influences of selected parameters of eddy current exciting system and of material thickness on the attenuation were studied by numerical means. A conductive plate specimen having the electromagnetic parameters of SUS316L was used for the study. A circular exciting coil positioned normally regarding the plate surface drove eddy currents. Three parameters of the exciting system, specifically the coil radius, the lift-off and the inspection frequency were altered together with the plate thickness. Distribution of eddy current density was evaluated for various adjustments of the parameters. The results clearly demonstrated that the coil radius and the lift-off as well have strong impact on the eddy current attenuation along material depth when a material thickness is comparable to the standard penetration depth.

\section{Acknowledgment}

This work was supported by the Slovak Research and Development Agency under the contracts No. APVV-0349-10 and APVV-0194-07. This work was also supported by grant of the Slovak Grant Agency VEGA, project No. 1/0765/11.

\section{References}

[1] RAO, B.P.C.: Practical eddy current testing. Alpha Science International Ltd.: Oxford, 2007.

[2] JANOUSEK, L., T. MAREK, T., D. GOMBARSKA, D.: Eddy Current Non-destructive Evaluation of Conductive Materials. Communications - Scientific Letters of the University of Zilina, 1, pp. 29-33, 2006.

[3] CHEN, Z., MIYA, K.: A New Approach for Optimal Design of Eddy Current Testing Probes. J. of Nondestructive Evaluation, vol. 17, pp. 105-116, 1998.

[4] STRAPACOVA, T., CAPOVA, K., JANOUSEK, L.: Artificial Heart Valve Inspection Using Eddy Current Techniques. Communications - Scientific Letters of the University of Zilina, vol. 13, No. 2A, pp. 83-86, 2011.

[5] JANOUSEK, L., SMETANA, M.: Uncertainty in Depth Evaluation of Partially Conductive Cracks from Eddy Current Testing Signals. Communications - Scientific Letters of the University of Zilina, vol. 13, No. 2A, pp. 55-60, 2011.

[6] SMETANA, M., STRAPACOVA, T.: Pulsed Eddy Currents as a New Approach in Non-destructive Evaluation of Conductive Materials. Proc. of TRANSCOM 2009, Zilina, pp. 63-66, 2009.

[7] GOMBARSKA, D., SMETANA, M.: Wavelet Based Signal Analysis of Pulsed Eddy Current Signals. Electrical review, vol. 87, pp. 37-39, 2011.

[8] MOTTL, Z.: The Quantitative Relations between True and Standard Depth of Penetration for Air-cored Probe Coils in Eddy Current Testing. NDT International, vol. 23, pp. 11-18, 1990.

[9] ALMAN, M.: Excitation Coil Parameters in View of Eddy Current Distribution in ECT Applications. Proc. of Elektro 2010, Zilina, pp. 221-224, 2010. 